\title{
FINITE ELEMENT ASSISTED METHOD TO REDUCE HARMONIC CONTENT IN THE AIRGAP FLUX DENSITY OF A HIGH TEMPERATURE SUPERCONDUCTING CORELESS ROTOR GENERATOR
}

\author{
B. Lukasik, K. F. Goddard and J. K. Sykulski \\ School of Electronics and Computer Science, University of Southampton, SO17 1BJ, United Kingdom \\ bl05r@ecs.soton.ac.uk, kfg@ecs.soton.ac.uk, jks@soton.ac.uk
}

Keywords: High temperature superconductivity, finite element analysis, design and optimisation.

\begin{abstract}
The paper reports on an investigation to reduce harmonic content in the airgap flux density of a high temperature superconducting synchronous generator with a coreless rotor. 3D finite element field simulation has been applied and a simple optimisation technique devised. The shape of specially designed flux diverters has been optimised.
\end{abstract}

\section{Introduction}

Recent advances in high temperature superconducting materials have opened new opportunities to build a new generation of power devices. However, a successful design needs to be preceded by careful modelling and optimization. This helps to identify possible problems and facilitates performance prediction. This paper reports on some of the challenges faced during the design of a synchronous generator with a coreless rotor which is under construction at the University of Southampton. Most problems are associated with the superconducting material used for the field winding. The BSCCO tape rated at 180A, used in the proposed design, is anisotropic and requires that the magnetic field, in particular its component perpendicular to the broad face of the tape, is kept below a certain value to avoid excessive reduction of the critical current. The design should also ensure that the harmonic content of the airgap flux is as low as possible.

\section{HTS coreless rotor design}

The rotor is designed to be of a coreless type, i.e. coils are not wound around an iron core, instead a magnetically neutral material is used. This approach poses a few significant difficulties. The lack of magnetic core greatly increases the magnetizing current needed to create a necessary air gap flux. It also increases the magnetic field in the superconductor. To alleviate the latter problem, the rotor is built as a stack of coils and flux diverters. The arrangement and the shape of the diverters are shown in Figure 1. The diverters are made of $9 \%$ nickel steal, proved to be the most suitable material for low temperatures and high saturation flux density devices, but mechanical considerations required the top hat, in the final design, to be made of Invar.
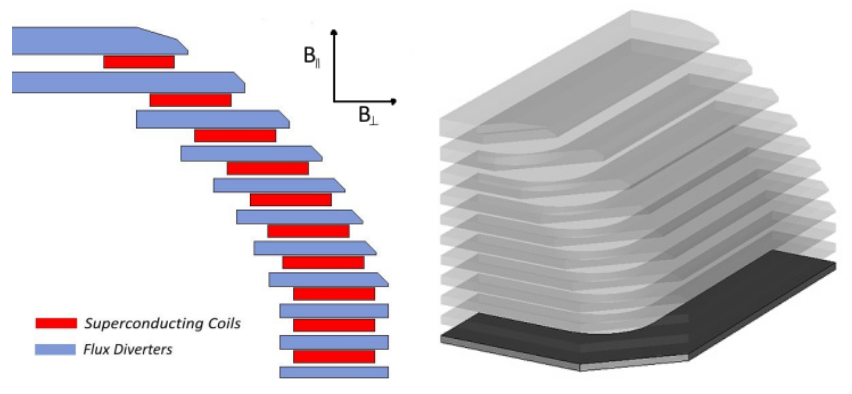

Figure 1. Initial rotor design. The stack of diverters and coils is shown on the left as a cross-section of the rotor. The picture on the right provides a 3D view (with only one eighth of the rotor shown due to symmetry).

\section{Electromagnetic considerations}

One of the main electromagnetic constraints in the design of the coreless rotor is that the magnetic field in the superconducting coils must not exceed acceptable limits. The limiting flux densities for the material used were $1.55 \mathrm{~T}$ for the field parallel to the broad face of the tape $\left(B_{\|}\right)$and $0.13 T$ for the perpendicular field $\left(B_{\perp}\right)$ both directions are indicated in Figure 1..The flux diverters are used to redirect the field in a way which ensures sufficient critical current in the superconductor. The shape of the top-most diverter has been chosen not only to divert the normal magnetic field from the coil but also to control the flux density waveform.

\section{Reducing harmonic content}

The harmonics content in the airgap flux density of the preliminary design shown in Figures 1 and 2a was too high and steps had to be taken to improve the waveform. This soon led, mainly because of the mechanical considerations, to the removal of the topmost coil and the two top diverters were replaced by one. Next an optimization procedure was introduced and - since the top diverter has the strongest influence on the waveform - most modifications were applied to that region of the rotor. The objective was to optimise the shape to minimize the harmonic content without unduly increasing the mass of the rotor. The best shape that has been found is shown in Figure $2 \mathrm{~b}$. Figure 4 confirms that this produces a significant drop mainly in the third harmonic. Unfortunately, mechanical considerations forced further changes, both in the proposed shape and the material used for the top hat, as described below. 

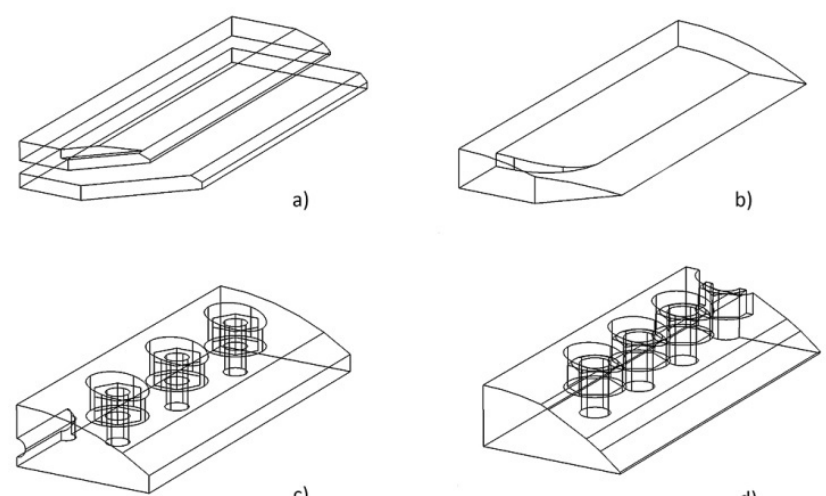

d)

Figure 2. Different shapes of the top-most diverter.

A set of clamping bolts had to be added. Initially it was assumed that 12 bolts would be enough, but then the number had to be increased to 14 . Moreover, since the toughness of $9 \%$ nickel steel is not guaranteed at temperatures below 73K, Invar has been chosen for the top hat mainly because of good mechanical properties at low temperatures. The shape is shown in Figure 2c.

Unfortunately the bolt holes increased the harmonic content in the airgap field. Since it was less obvious which region of the top-most diverter should be changed to improve the waveform, an attempt was made to estimate the effect of changing the local flux density on the total harmonic content of the voltage waveform by implementing a simple procedure first suggested in [1], by first using

$$
\frac{\partial V_{i}^{2}}{\partial B_{r}(\theta)}=2 V_{i} \frac{\partial V_{i}}{\partial B_{r}(\theta)}
$$

where $V_{i}$ is the $i$-th voltage harmonic and $B_{r}$ is the radial flux density, and then applying the quotient rule. The formula helps to identify areas where the local flux density should be changed to influence the harmonic content of the output waveform. Applying this rule to the given voltage waveform yields the graph shown in Figure 3. The curve shows the contribution of the local flux density to the total harmonic content of the voltage waveform. It implies that the airgap flux density could be reduced in the region around the angle of $70^{\circ}$ where the contribution of harmonics is high. The reduction is actually achieved mainly through a series of chamfers in the top-most diverter. These are clearly visible in Figures $2 c$ and $2 d$. Whereas the graph also shows that it would be desirable to increase the flux in the region around the angle of $15^{\circ}$, this could not be achieved without sacrificing preferred objectives (e.g. weight reduction), since it would mean adding more magnetic material into the body of the rotor.

The differences between various shapes in terms of content of harmonics are demonstrated in Figure 4. It shows that the content of harmonics has been reduced by using the above rule without complicating the shape of the top hat.

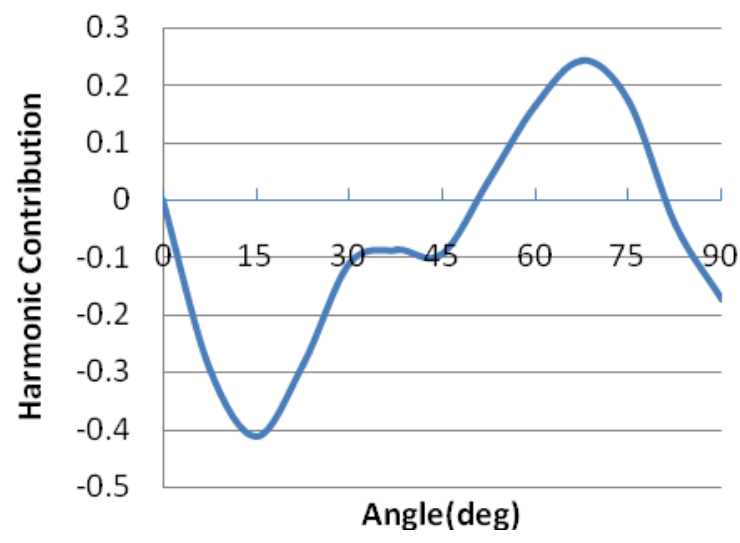

Figure 3. Contribution of local flux density to the total harmonic content of the voltage waveform.

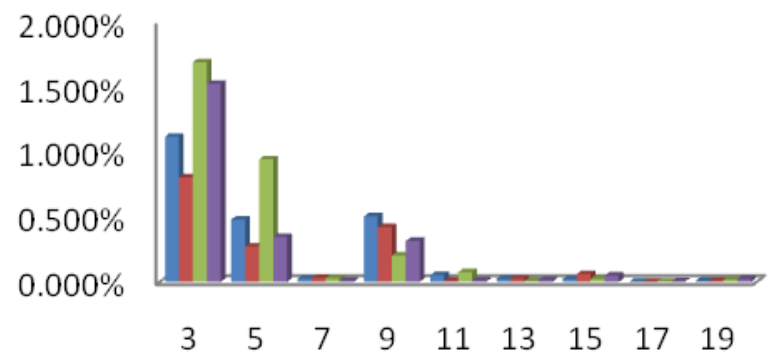

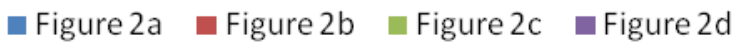

Figure 4. Percentage harmonic content for diverter shapes presented in Figure 2.

\section{Conclusions}

The paper demonstrates the complexity of the design of a superconducting machine with a coreless rotor. 3D electromagnetic finite element modelling has been found useful in achieving design objectives. The content of harmonics in the voltage waveform was successfully reduced by using a simple optimisation procedure.

\section{Acknowledgements}

This work is sponsored by the EPSRC (UK), grant number EP/D000688/1.

\section{References}

[1] K. S. Ship, J. K. Sykulski and K. F. Goddard. "Field optimisation in a synchronous generator with high temperature superconducting field winding and magnetic core", IEE Proc.-Sci. Measur. Technol., 149(5), pp. 194-198, (2002).

[2] K. S. Ship and J. K. Sykulski. "Field simulation studies for a high temperature superconducting synchronous generator with a coreless rotor", IEE Proc.-Sci. Meas. Technol., 151(6), pp. 414-418, (2004). 